\title{
Must potential pain mean category E?
}

Dr. Raj Subaraman, a noted neurophysiologist, recently moved his lab to Great Eastern University and submitted a protocol using the spared nerve injury model to the Great Eastern IACUC. Subaraman proposed to anesthetize his rats and then expose the sciatic nerve at the point where it trifurcated into the tibial, common peroneal and caudal sural nerves. The first two nerves were transected but the sural nerve was left intact. Subaraman's research team had done the procedure many hundreds of times previously, and he was able to assure the IACUC that there would be no motor dysfunction in the operated limb. By that he meant that the rat would be able to walk and groom normally and also gain weight normally. In fact, during the first postoperative week, abnormalities (such as increased sensitivity to heat or cold) were found only when specific neurological tests were carried out.
Based on his past experience, Subaraman knew that about one week after surgery, some animals would begin to show clinical signs of neuropathy (nerve degeneration, often with associated pain), and so he routinely began his experimental treatments-the crux of his research-four days after surgery. When the treatments were successful, neuropathy and pain were quite minimal and transient. However, when the Great Eastern IACUC finally approved Subaraman's protocol, it was with the condition that all of the operated animals would be placed in USDA pain or distress category E (unalleviated pain or distress). Great Eastern University, like many other schools, used the USDA's pain and distress categories even though it was not required to do so. At Subaraman's former institution, rats that had undergone surgery but had no (or very minimal) motor dysfunction at any time were placed in category D (alleviated pain or distress) as a consequence of the required surgical intervention. Only those animals that developed clinically apparent neuropathy were retroactively placed in category E. Subaraman thought that was fair, but the Great Eastern IACUC said that because the specific neurological tests were able to detect hypersensitivity to heat or cold during the first few postoperative days, there was a reasonable potential for pain or distress to be present on a continuous or near continuous basis. Although Subaraman argued, it was to no avail. The IACUC would not budge.

What is your opinion? Should all of Subaraman's rats be placed in category E, even though increased pain sensitivity is recognized only when the condition is artificially provoked by cold or heat, or is it more appropriate to have clinically normal animals in category D and only those with clinical neuropathy in category $\mathrm{E}$ ?

\section{RESPONSE}

\section{Much ado about nothing!}

\section{William R. Parlett Jr., DVM, DACLAM}

Classify as category D or E? Does it matter to the rats or to USDA? No. For any given manipulation of the rats, the pain category classification will have no impact on them whatsoever. The USDA regulations exclude rats of the genus Rattus that are bred for research. So we can assume that the IACUC's policy is simply to apply the same requirements to all speciesa laudable, even if unnecessary, goal.

It matters not whether pain is artificially provoked or unprovoked; it is pain either way. Whether hypersensitivity to cold or heat is painful could be argued, depending on degree of the cold or heat, the duration of exposure and the ability of the animal to freely move away from the source of the cold or heat. Given that at Subaraman's former institution, only the animals that experienced clinical neuropathy were placed in category E, we can assume that the hypersensitivity, and its detection, in this case does not involve more than slight or momentary discomfort-that is, it is not significant.

Because some of the rats will eventually be classified as category E, and because Great Eastern is not excluding rats from the requirements applicable to USDAcovered species, Subaraman must provide scientific justification that the pain or distress cannot be alleviated. We can assume that Subaraman has acknowledged that some of the rats will experience unalleviated pain or distress and has provided the justification and that the IACUC accepted the justification. Therefore, the procedures are approvable.
The only questions are which pain category the rats should be assigned to and the timing of these assignments.

Based on Subaraman's experience, it is known that some of the rats will experience unalleviated pain or distress. This number or percentage of the total number of rats should be classified in category E from the start. Which position would be preferable to defend: (i) that the rats were initially classified as category $\mathrm{D}$ but should have been in category $\mathrm{E}$ or (ii) that the rats were initially classified as category E but, based on close clinical evaluation, did not experience unalleviated pain and could be classified as category $\mathrm{D}$ ?

I suggest the following solution: initially, all the rats should be placed in category E. Close post-operative observation and evaluation by research, veterinary and husbandry staff members 